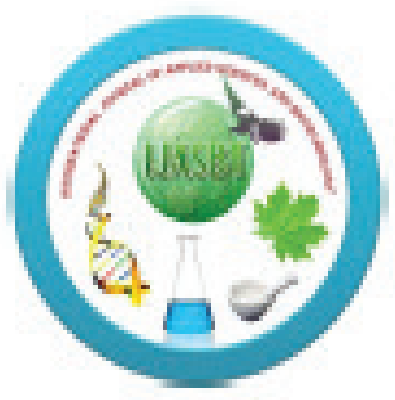

OPEN ACCESS

Avallable online at wwwijastot.org

International Journal of Applied Sciences and Biotechnology A Rapid Publishing Journal

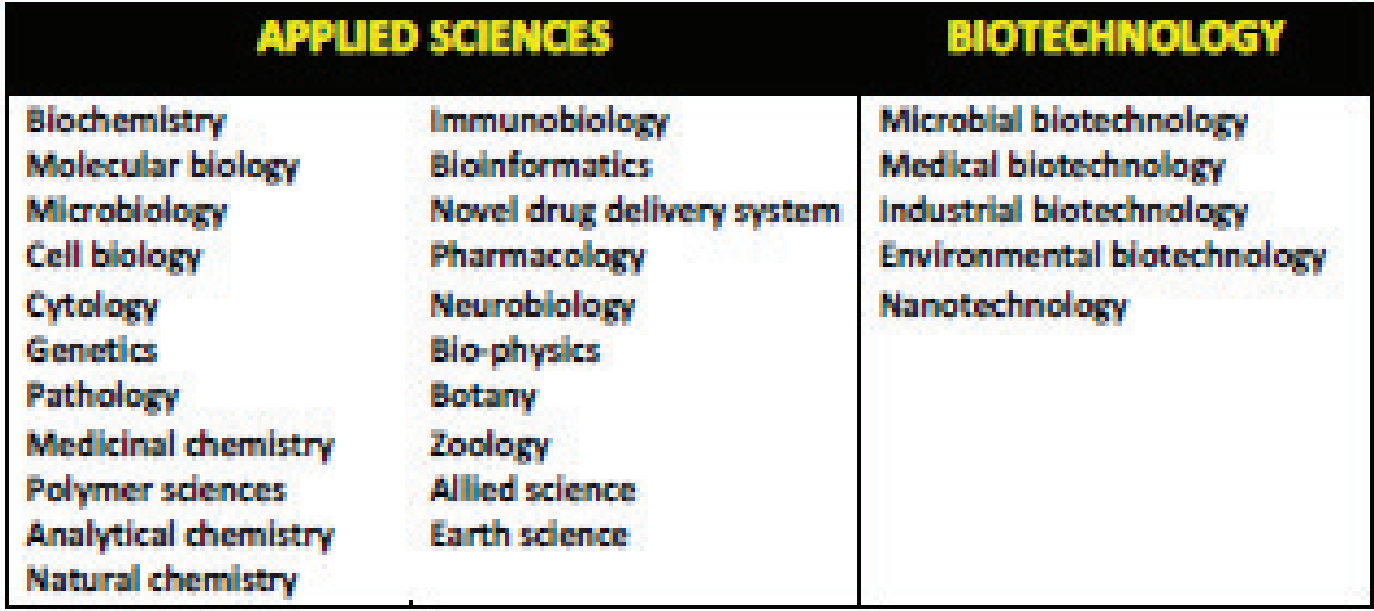

If any queries or feedback, then don't hesitate to mail us at: editorijasbtiegmail.com 


\title{
COMPARATIVE GENOMIC STUDIES AND IN-SILCO STRATEGIES ON LEISHMANIA BRAZILENSIS, LEISHMANIA INFANTUM AND LEISHMANIA MAJOR: CONSERVED FEATURES, PUTATIVE FUNCTIONS AND POTENTIAL DRUG TARGET
}

\author{
Rakesh N R*1, Pradeep S
}

\begin{abstract}
${ }^{1}$ Department of Biotechnology, KLE Dr M S Sheshgiri College of Engineering and Technology, Belgaum- 590008, Karnataka. ${ }^{2}$ Department of Biotechnology, BMS College Of Engineering, Bull Temple Road, Bangalore- 560019 Karnataka.

*Corresponding Author: rs19neelgund@gmail.com
\end{abstract}

\begin{abstract}
Leishmaniasis is a parasitic disease found largely in the tropics, which the World Health Organization has estimated infects 12 million people worldwide each year. More recently cases have been reported in Europe among intravenous drug users with HIV. At least 20 Leishmania species infect humans. New world parasite Leishmania. braziliensis is the causative agent of mucocutaneous Leishmaniasis. The old world species Leishmania. major and Leishmania. infantum, which are present in Africa, Europe and Asia, are parasites that cause cutaneous and visceral Leishmaniasis respectively. Aim of this Study is determination of major common genes and Protein identified Gene location on each of the chromosomes, and identification of a common protein drug target Promastigote surface antigen with available lead molecule acetylglucosamine (6-(acetylamino)-6-deoxyhexopyranose) and docking studies on those considered Leishmania species.
\end{abstract}

Keywords: Leishmaniasis, Promastigote surface antigen, Acetylglucosamine

\section{Introduction}

Leishmaniasis is a parasitic disease found largely in the tropics, which the World Health Organization has estimated infects 12 million people worldwide each year. More recently cases have been reported in Europe among intravenous drug users with HIV. At least 20 Leishmania species infect humans. New world parasite Leishmania. braziliensis is the causative agent of mucocutaneous Leishmaniasis. The old world species Leishmania. major and Leishmania. infantum, which are present in Africa, Europe and Asia, are parasites that cause cutaneous and visceral Leishmaniasis respectively (Christopher S Peacock et al, 2007). Three type of Leishmaniasis Visceral Leishmaniasis: The most serious form in which parasites leave the inoculation site and proliferate in liver, spleen and bone marrow, resulting in host immuno-suppression and ultimately death in the absence of treatment. Cutaneous Leishmaniasis: In which parasites remain at the site of infection and cause localized long-term ulceration.
Mucocutaneous Leishmaniasis: A chronic destruction of mucosal tissue that develops from the cutaneous disease in less than $5 \%$ of affected individuals.

In India Visceral Leishmaniasis (VL), also known as kala-azar, black fever, and Dumdum fever is the most severe form of Leishmaniasis. (Lira R, Sundar S, Makharia A, Kenney R, Gam A, et al, 1999)Leishmaniasis is a disease caused by protozoan parasites of the Leishmania genus. This disease is the second-largest parasitic killer in the world (after malaria), responsible for an estimated 500,000 infections each year worldwide (William D, Berger, Timothy G et al, 2006). Diagnosis for each of these types of Leishmaniasis involves taking a scraping from a lesion, preparing it in a laboratory, and examining it under a microscope to demonstrate the causative protozoan with a skin test (similar to that test for TB). Phlebotomine sand flies are the only known natural vectors of Leishmania more than 400 Phlebotomine species are described fewer than fifty are known to be involved in the transmission cycle of these parasites some vectors species are highly 
restricted to the species of Leishmania that they transmit in nature.

The objective of our study was determination of major common genes and protein identified gene location on each of the chromosomes, and identification of a common protein drug target Promastigote surface antigen with available lead molecule acetylglucosamine (6-(acetylamino)-6deoxyhexopyranose) and docking studies on those considered Leishmania species. (Marsden, P.D. Mucosal Leishmaniasis, et al, 1986)

\section{Material and Methods}

Species for the genomic studies and their in-silco strategies

\section{Leishmania. major Friedlin}

Leishmania.major, the reference strains (MHOM/IL/80/Friedlin, zymodeme MON-103) it was first sequenced as part of a multi-centre collaboration. The genome has been manually annotated and so far more than 8,000 genes have been identified in the $\sim 33.6 \mathrm{Mb}$ haploid genome which is spread over 36 chromosomes the database is undergoing continual manual annotation and citation.

\section{Leishmania infantum}

The genome of Leishmania. infantum is the second Leishmania species to be sequenced at the Sanger Institute. The clone used was generated a whole genome shotgun sequence of Leishmania. infantum JPCM5 (MCAN/ES/98/LLM-877). This pathogen is the causative agent of visceral Leishmaniasis in the Mediterranean basin. Gene prediction and annotation has been partly generated and analyzed by comparison to the Leishmania. major genome as sequencing and annotation are in progress, the data is continually updated.

\section{Leishmania. braziliensis}

Leishmania. braziliensis the third Leishmania species to be sequenced at the Welcome Trust Sanger Institute. Responsible for causing localized cutaneous lesions in Table 1: In-silco Genome strategies of all the three species of Leishmania

\begin{tabular}{llll}
\hline Genomic Attributes & Leishmania major & Leishmania infantum & Leishmania. braziliensis \\
\hline Chromosome number & 36 & 36 & 35 \\
Size( bp) & $32,816,678$ & $32,134,935$ & $32,005,207$ \\
Overall G+C content (\%) & 59.7 & 59.3 & 57.76 \\
Coding genes & 8,298 & 8,154 & 8,153 \\
Pseudo genes & 97 & 41 & 161 \\
\hline
\end{tabular}

affected people. Leishmania. braziliensis can also result in a more destructive, progressive infection in mucosal tissue Karyotyping of Leishmania. braziliensis has shown that it has 35 chromosomes compared to the 36 present in old world species. The difference in chromosome number is due to the fusion of chromosomes 20 and 34 to make a single chromosome in Leishmania. braziliensis.

\section{Results and Discussion}

\section{Summary of Genomes:}

The genomic studies and their in-silco strategies of all the three species of Leishmania been shown in the Table 1

\section{Common Genes and there locus:}

One of the studies involved in the insilico-detection of common genes and their locus on all the chromosomes with the three Leishmania species is been tabulated from chromosome number 1 to chromosome number 36. An example or sample of common genes located on the chromosome 1 and 2 is shown in table 2 and table 3 respectively.

\section{Common Protien target in all the three species of Leishmania:}

PSA (Promastigote surface antigen) is one of the major classes of membrane proteins present at the surface of the parasitic protozoan Leishmania. (El- Sayed, N.M. et al, 2005). PSA is a Leishmania family of membranebound or secreted proteins. Its Basic Local Alignment Search was done against NCBI's Genbank Database and Multiple Sequence Alignment was carried out using ClustalW an online multiple alignment tool. The below figure 1 shows the result of the Protein BLAST in all three Leishmania and figure 2 shows the alignments of the Promastigote surface antigen sequence with three Leishmania species. (Croft SL, Coombs GH. et al, 2003) 
Rakesh NR and Pradeep S (2013). Int J Appl Sci Biotechnol, Vol. 1(2): 62-66

Table 2: Common Genes and there locus on Chromosome Number 1

\begin{tabular}{llll}
\hline Product Name & Leishmania major & $\begin{array}{l}\text { Leishmania } \\
\text { infantum }\end{array}$ & $\begin{array}{l}\text { Leishmania. } \\
\text { braziliensis }\end{array}$ \\
\hline phosphoglycan beta 1,3 galactosyltransferase 3 & LmjF02.0010 & LinJ02.0140 & LbrM02_V2.0010 \\
cytochrome b-domain protein, putative & LmjF02.0050 & LinJ02.0020 & LbrM02_V2.0070 \\
exportin T (tRNA exportin)-like protein & LmjF02.0110 & LinJ02.0090 & LbrM02_V2.0120 \\
phosphatidylinositol 3-kinase-like protein & LmjF02.0120 & LinJ02.0100 & LbrM02_V2.0130 \\
small GTP binding protein rab6-like protein & LmjF02.0260 & LinJ02.0180 & LbrM02_V2.0290 \\
protein kinase, putative & LmjF02.0290 & LinJ02.0210 & LbrM02_V2.0340 \\
ABC1 transporter, putative & LmjF02.0300 & LinJ02.0220 & LbrM02_V2.0350 \\
casein kinase II, alpha chain, putative & LmjF02.0360 & LinJ02.0280 & LbrM02_V2.0390 \\
proteasome regulatory non-ATPase subunit 6, putative & LmjF02.0370 & LinJ02.0290 & LbrM02_V2.0400 \\
FtsJ-like methyltransferase, putative & LmjF02.0380 & LinJ02.0300 & LbrM02_V2.0410 \\
ubiquitin-conjugating enzyme e2, putative & LmjF02.0390 & LinJ02.0310 & LbrM02_V2.0420 \\
RNA-editing complex protein MP81, putative & LmjF02.0410 & LinJ02.0330 & LbrM02_V2.0430 \\
protein kinase, putative & LmjF02.0570 & LinJ02.0490 & LbrM02_V2.0540 \\
ARP2/3 complex subunit, putative & LmjF02.0600 & LinJ02.0520 & LbrM02_V2.0580 \\
gamma-glutamyl phosphate reductase-like protein & LmjF02.0630 & LinJ02.0550 & LbrM02_V2.0610 \\
mitochondrial carrier protein, putative & LmjF02.0670 & LinJ02.0590 & LbrM02_V2.0650 \\
metallo-peptidase, Clan MA(E), Family M3 & LmjF02.0740 & LinJ02.0660 & LbrM02_V2.0670 \\
\hline
\end{tabular}

Table 3: Common Genes and there locus on chromosome number 2 as an example

\begin{tabular}{llll}
\hline Product Name & Leishmania major & $\begin{array}{l}\text { Leishmania } \\
\text { infantum }\end{array}$ & $\begin{array}{l}\text { Leishmania. } \\
\text { braziliensis }\end{array}$ \\
\hline phosphoglycan beta 1,3 galactosyltransferase 3 & LmjF02.0010 & LinJ02.0140 & LbrM02_V2.0010 \\
cytochrome b-domain protein, putative & LmjF02.0050 & LinJ02.0020 & LbrM02_V2.0070 \\
exportin T (tRNA exportin)-like protein & LmjF02.0110 & LinJ02.0090 & LbrM02_V2.0120 \\
phosphatidylinositol 3-kinase-like protein & LmjF02.0120 & LinJ02.0100 & LbrM02_V2.0130 \\
small GTP binding protein rab6-like protein & LmjF02.0260 & LinJ02.0180 & LbrM02_V2.0290 \\
protein kinase, putative & LmjF02.0290 & LinJ02.0210 & LbrM02_V2.0340 \\
ABC1 transporter, putative & LmjF02.0300 & LinJ02.0220 & LbrM02_V2.0350 \\
casein kinase II, alpha chain, putative & LmjF02.0360 & LinJ02.0280 & LbrM02_V2.0390 \\
proteasome regulatory non-ATPase subunit 6, putative & LmjF02.0370 & LinJ02.0290 & LbrM02_V2.0400 \\
FtsJ-like methyltransferase, putative & LmjF02.0380 & LinJ02.0300 & LbrM02_V2.0410 \\
ubiquitin-conjugating enzyme e2, putative & LmjF02.0390 & LinJ02.0310 & LbrM02_V2.0420 \\
RNA-editing complex protein MP81, putative & LmjF02.0410 & LinJ02.0330 & LbrM02_V2.0430 \\
protein kinase, putative & LmjF02.0570 & LinJ02.0490 & LbrM02_V2.0540 \\
ARP2/3 complex subunit, putative & LmjF02.0600 & LinJ02.0520 & LbrM02_V2.0580 \\
gamma-glutamyl phosphate reductase-like protein & LmjF02.0630 & LinJ02.0550 & LbrM02_V2.0610 \\
mitochondrial carrier protein, putative & LmjF02.0670 & LinJ02.0590 & LbrM02_V2.0650 \\
metallo-peptidase, Clan MA(E), Family M3 & LmjF02.0740 & LinJ02.0660 & LbrM02_V2.0670 \\
\hline
\end{tabular}

\begin{tabular}{|l|l|l|}
\hline \multicolumn{5}{|c|}{ Distribution of 102 Blast Hits on the Query Sequence } \\
\hline Mouse over to see the defline, click to show alignments \\
\hline
\end{tabular}

Fig. 1: The result of the Protein BLAST against NCBI's GenBank Database 
Rakesh NR and Pradeep S (2013). Int J Appl Sci Biotechnol, Vol. 1(2): 62-66

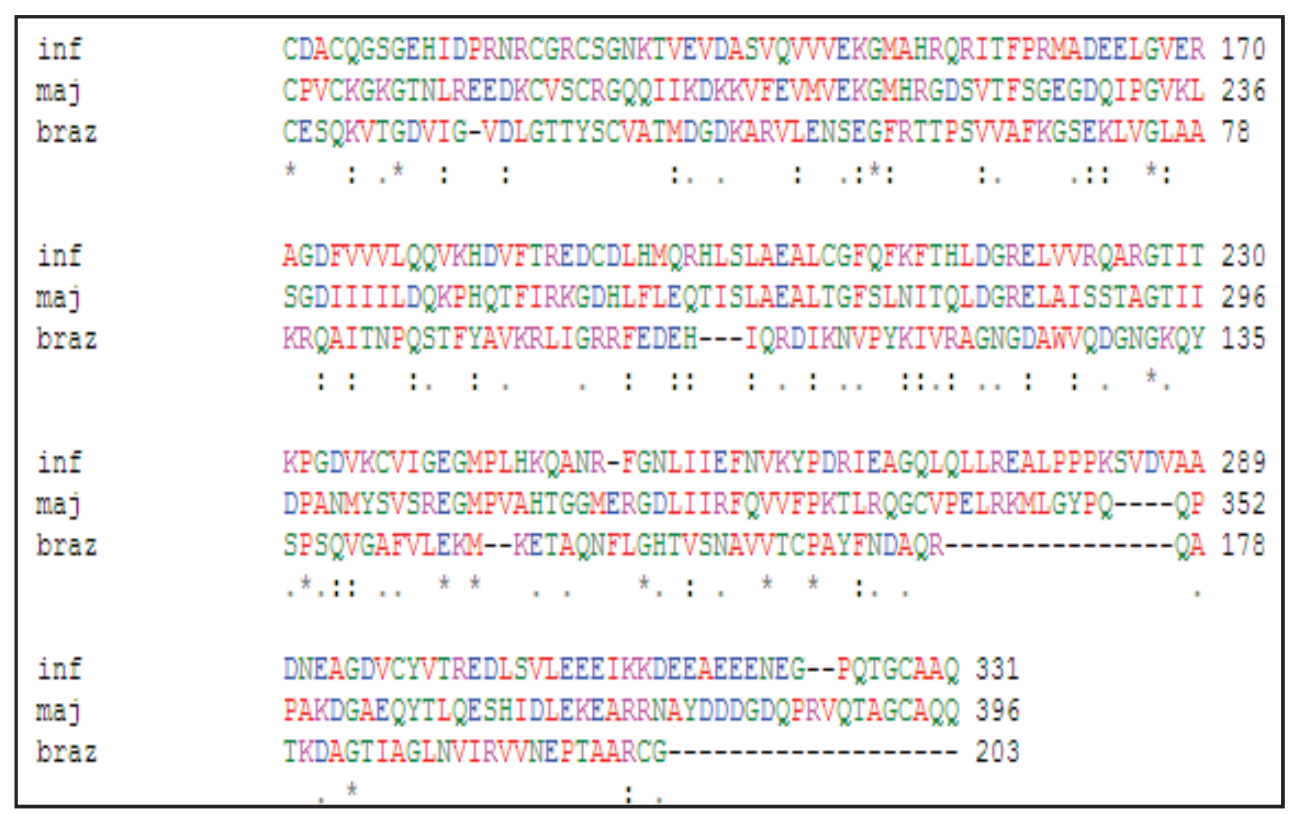

Fig. 2: Multiple sequence alignment of Promastigote surface antigen conserved regions with three Leishmania species.

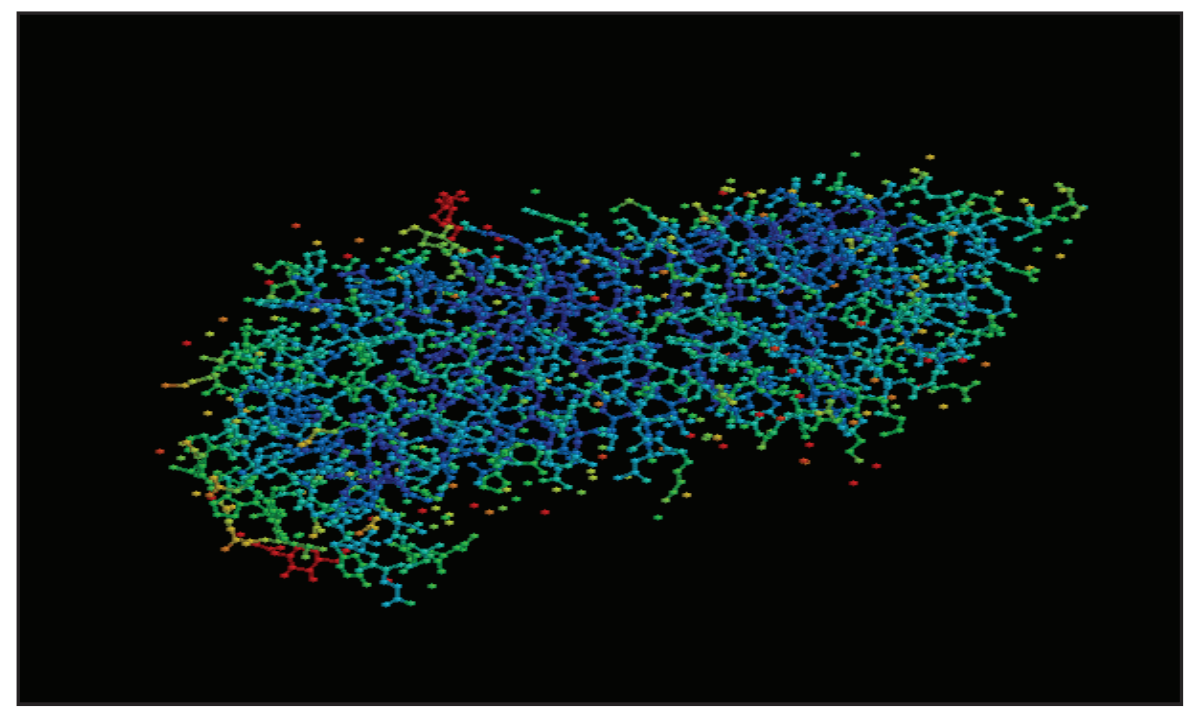

Fig. 3: The Ball and Stick 3-Dimensional view of Promastigote surface antigen (PSA) in Arguslab workspace window

\section{Docking Results:}

Clustering the final poses $=44$ final unique configurations. Number of local searches that succeeded in locating new minims $=3$. Re-clustering the final poses $=44$ final unique configurations. Best Ligand Pose: energy $=-5.56111 \mathrm{kcal} / \mathrm{mol}$. Docking run: elapsed time $=11$ seconds.

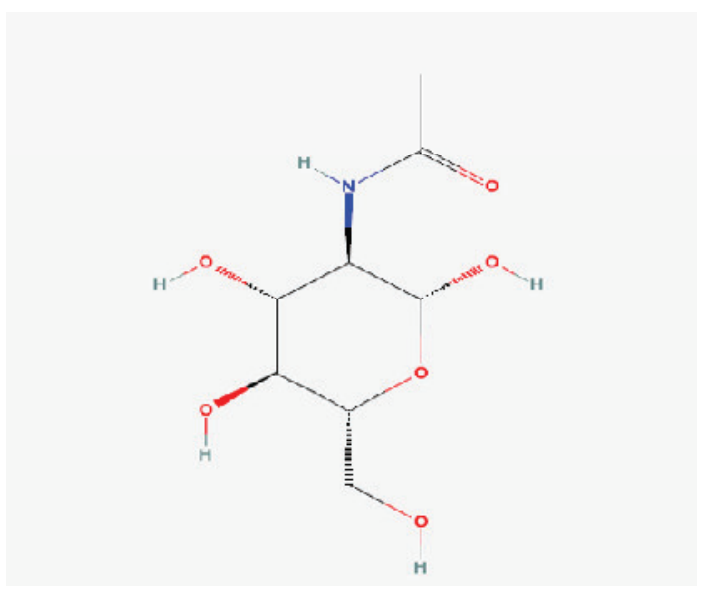

Fig. 4: Two-Dimensional structure of the Inhibitor Acetylglucosamine: (6-(acetylamino)-6deoxyhexopyranose) 


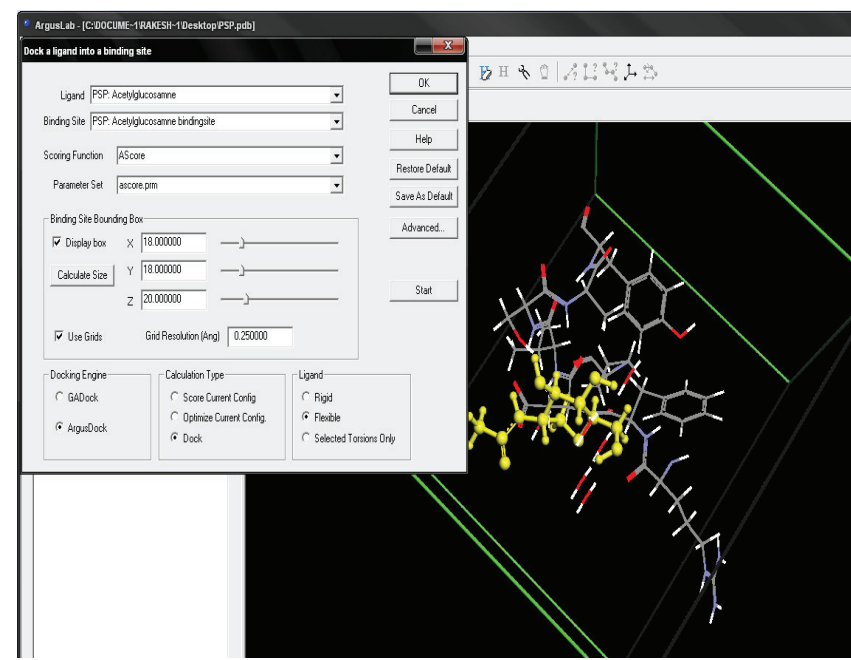

Fig. 5: Optimization of the Ligand and its Binding site in Arguslab with grid setting.

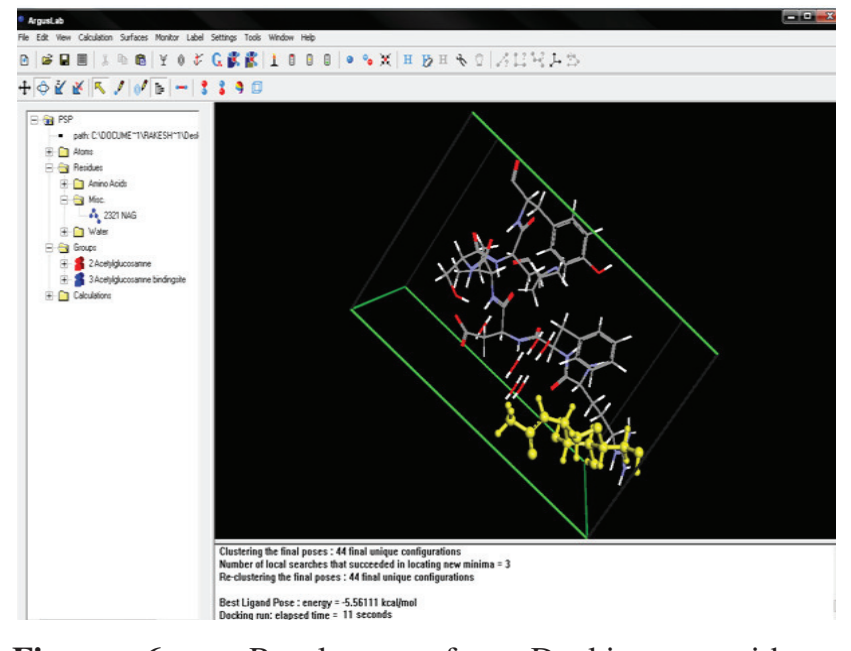

Fig. 6: Results of Docking with Ligand(Acetylglucosamine) and Receptor (Promastigote surface antigen) with elapsed time and best ligand pose energy.

\section{Conclusion}

Comparisons of the complete genomes of three species of Leishmania allowed revealing a novel genus-specific gene. A common drug target was identified within 3 species. Identification of a few genes that are either species-specific or under positive selective pressure provides a comprehensive and manageable resource to target efforts in identifying parasite factors that influence infection. Conversely, factors that are unique to the Leishmania genus but common to all species may be used as potential drug targets or vaccine candidates.

\section{Acknowledgments}

The authors are thankful to the Dr. S. C. Pilli, Principal, KLE and Dr. M.S. Sheshgiri College of Engineering and Technology, Udyambag, Belgaum for providing healthy environment for this work. We express our immense gratitude to our beloved HOD Dr. S. C. Mali for his support and suggestions to carry the above work and giving permission to publish the results.

\section{References}

Christopher S Peacock et al. (2007) Comparative genomic analysis of three Leishmania species that cause diverse human disease. Nature Genetics 39, 839 - 847.

Croft SL, Coombs GH. (2003) Leishmaniasis-current chemotherapy and recent advances in the search for novel drugs. Trends Parasitol. 19, 502-508.

El- Sayed , N.M. et al. (2005). Comparative genomics of trypanosomatid parasitic protozoa. Article Pub Med ISI ChemPort 309, 404-409.

James, William D Berger, Timothy G et al. (2006). Andrews diseases of the Skin: clinical dermatology. Saunders Elsevier. ISBN 0- 7216-2921.

Lira R, Sundar S, Makharia A, Kenney R, Gam A, Saraiva E, Sacks D. (1999) Evidence that the high incidence of treatment failures in Indian kala-azar is due to the emergence of antimony-resistant strains of Leishmania donovani. J. Infect. Dis. 180, 564-567.

Marsden, P.D. Mucosal Leishmaniasis, Trans. R. Soc. Trop. (1986). Article Pub Med ChemPort 80, 859-876. 